\title{
Improved technology for manufacture of carbon electrodes
}

\author{
A PLATON, A DUMBRAVA*,+, N IUTES-PETRESCU and \\ LUIZA SIMIONESCU \\ National Research Institute for Petroleum Processing and Petrochemistry \\ (INCERP), Boulevard Republicii no. 291A, 2000 Ploiești, Romania \\ +Present address: 205, 1039-13 Avenue, SW, Calgary, Alberta T2R OL5, \\ Canada \\ e-mail: adumbrava@hotmail.com; iutes@serv.incerp.ro
}

MS received 16 October 1999; revised 1 December 1999

\begin{abstract}
Current industrial carbon electrodes are typically manufactured by blending petroleum coke particles (the filler) with molten coal tar pitch (the binder) and extruding the resultant mix to form the 'green electrode'. This is then baked under controlled conditions. In case of usage as anodes in steel electric furnaces (or as other carbon and graphite products), the electrodes could undergo further processing like pitch impregnation or graphitization. During heat treatment, some of the organics are destructively distilled, vaporized or decomposed, resulting in carbon deposition in the electrode. As the vaporized materials exit the body of the electrode they cause porosity in the walls, which results in reduction in density, current carrying capacity and flexural strength.

The paper presents investigations to improve some physico-chemical characteristics of these electrodes (such as coefficient of thermal expansion, mechanical strengths, density, pore volume, porosity etc.), obtained in different manufacture steps, by addition of varieties of coal tar pitch. These include attempts to improve the chemical compatibility of the coke-pitch system in the mixture and establish the method and the point of introduction of additive, the concentration required and appropriate analytical control during the entire manufacture. Methods of analysis used include thermogravimetry and porosimetry. The microstructure of the electrodes is investigated through a wide range and the data obtained include pore size and pore volume distribution, surface area, porosity, particle size distribution and type of pores. The overall results clearly indicate better characteristics and performance for electrodes with additives as against electrodes without them, such as lower porosity, lower thermal expansion coefficients and greater mechanical strength. These data are analyzed with respect to the process step and electrode type.
\end{abstract}

Keywords. Electrode characteristics; petroleum coke; porosity; coal tar pitch.

\section{Introduction}

Although petroleum coke is a by-product of oil refining, it finds broad usage in many chemical and metallurgical processes. Its main application is its significant use in

*For correspondence 
aluminium and steel industries (carbon electrode manufacture), chemical industry (titanium dioxide preparation) etc.

Commercially, electrodes up to $700 \mathrm{~mm}$ in diameter and up to $3 \mathrm{~m}$ in length are produced for utilization in electric arc furnaces. These are used to carry large quantities of electricity in the steel melting process. They have to possess a series of characteristics such as: (i) high density, (ii) high modulus of elasticity, (iii) high electrical conductivity, (iv) high mechanical strength, and (v) improved oxidation resistance.

Our research project initiated a few years ago is dedicated to improvement of some characteristics such as density, mechanical strength, coefficient of thermal expansion, porosity etc. by combined chemical and technological means.

The main research targets of the investigation include determination of the following:

- improvement of chemical compatibility in the calcined petroleum coke-pitch system in the mixture by addition of coal tar pitch ${ }^{1,2}$;

- the decision regarding when to introduce the additive in the manufacturing sequence;

- concentration range of additive to be introduced;

- analytical control of the electrodes in all steps of the manufacturing cycle;

- commercial trials with the electrodes, based on the latest technological methods.

The present method has been applied and checked with both standard and needle coke containing carbon electrodes.

\section{Technological background}

Petroleum coke is used in carbon electrode manufacture and is subjected to severe quality criteria for its purity and physico-chemical properties. In conventional technologies, calcined premium coke particles (the filler) obtained from the retarded coking process are mixed with coal pitch (the binder). The resulting mixture is extruded to desirable dimensions (length, diameter) to form the so-called 'green' electrodes ${ }^{3}$.

These electrodes are then baked at elevated temperature, under controlled conditions of time duration, temperature gradient and temperature level. This heat treatment (performed at approximately $1300^{\circ} \mathrm{C}$ ) enhances the carbon content of the green electrode from approximately $95 \%$ to greater than $99 \%$, by decomposition, distillation or vaporisation of part of the organic material. The vaporised materials escape through the body of the electrode, leaving behind a porous structure. The result of this inherent porosity is reduction in the density, mechanical strength and current-carrying capacity of the material. In order to reduce this inherent porosity, the electrodes used in steel electric arc furnaces as well as in other products have to undergo further heat treatment and graphitization. First, the electrodes are impregnated with a variety of pitch which must have physico-chemical properties suitable for this purpose. On heating the carbon to temperatures between 2700 and $3000^{\circ} \mathrm{C}$, its crystalline structure is completely modified and results in a structure characterized by low resistivity, high thermal conductivity, improved oxidation resistance, high strength etc.

The compatibility of petroleum coke with pitches is defined as the capacity of the fluid system (pitch) to aggregate a certain amount of solid (coke) in a compact structure. This compatibility is a complex function dependent on a variety of factors such as temperature, solid granulation, nature of the solid surface, adhesion strength, stress type and size, applied physico-chemical treatments etc. ${ }^{4}$. 
Generally, the coke-pitch system compatibility can be estimated by measuring the wetting angle which characterizes the solid-liquid adhesion size. We have developed a new method to quantify the degree of compatibility of the coke-pitch system by the ratio

$$
\Delta=\text { coke mass } / \text { coal tar mass. }
$$

Experimentally this dependence can be determined by a complex function using the relation.

$$
\Delta=\Delta(T, C, \phi, \eta, \sigma),
$$

where $T$ is the temperature, $C$ the chemical composition, $\phi$ the particle size distribution, $\eta$ the dynamic viscosity, and $\sigma$ the surface tension.

This method was applied successfully to adjust the chemical compatibility between coke (acidic) and coal tar pitch (basic). This unique combination of physical and chemical properties makes carbon and graphite electrodes very suitable for a variety of applications.

\section{Experimental}

Experiments have been carried out by the addition of coal pitch, in the manufacture of carbon electrodes up to $600 \mathrm{~mm}$ in diameter and $2200 \mathrm{~mm}$ in length. The addition can be done in two ways:

(a) addition at ambient temperature (dry aggregate step), and

(b) addition at elevated temperature (paste preparation step).

The procedures have been carried out using controlled additive concentrations. Some of the physico-chemical properties of the electrodes (both with and without additives) have been controlled at every major step of the manufacture as below.

(i) Formation of the green electrodes.

(ii) Baking of the electrodes.

(iii) Impregnation and rebaking of the electrodes.

(iv) Graphitization of the electrodes.

\section{Analytical control}

Analytical control has included different methods such as:

- thermal analysis (DTA and DTG),

- porosimetry,

- optical microscopy.

Since these methods consider small amounts of matter, special attention has been paid to the point from where the electrode sample has been drawn. Difference in structure between the core and the exterior zones of the electrode has been assumed. Consequently, 
samples have been drawn from three different zones: A - core zone; B - medium zone; $\mathrm{C}$ - external zone.

\subsection{Thermal analysis method}

Various components of any complex material interact differently. These interactions determine the corresponding energy levels of differing magnitudes (illustrated by the differential thermal analysis, DTA, curve). Different locations and shapes of the peaks are affected by composition and structural changes. At the same time, the volume of interaction is responsible for the loss of mass owing to the exit of volatile compounds, which is a function of the temperature and the nature of electrode type (illustrated by the DTG curve).

Determinations are made in an argon atmosphere, using constant rate of heating $\left(10^{\circ} \mathrm{C} / \mathrm{min}\right)$.

\subsection{Porosimetry method}

Study of the texture of analysed samples was carried out on a Porosimeter 400 type apparatus (Carlo Erba). Using two physical principles - gas adsorption and mercury penetration - the physical microstructure of any solid material sample can be investigated over a large range of porosities (for pore radii between 0.5 and $6 \times 10^{5} \mathrm{~nm}$ ). A computer controls all the steps of the operation for maximum operating efficiency and provides the calculated and recorded data. The apparatus provides pressures of up to $4 \times 10^{8} \mathrm{~N} / \mathrm{m}^{2}$.

We can determine the following parameters:

- pore size distribution,

- pore volume, size and distribution,

- specific surface area,

- particle size distribution.

In addition, qualitative information on the shape and the type of pores can be determined. For this study, the pore size distribution has been split into the following regions:

- micropores: 0-300 $\mathrm{A}$,

- macropores: $300-100,000 \AA$,

- supermacropores: $100,000 \AA$.

\section{Results and discussions}

\subsection{Green electrodes}

Figure 1 presents the plots of energy levels versus temperature for the following situations:

NA - electrode without additive,

CA - (cold) electrode with additive,

HA1 - (hot) electrode with additive (additive concentration C1),

HA2 - (hot) electrode with additive (additive concentration C2). 
DTA curves highlight the endothermic effect of structural changes occurring during heating over a large range of temperature $\left(20-800^{\circ} \mathrm{C}\right)$. The largest thermal effects are recorded for electrodes with additives especially for CA and HA2 electrodes. The data show major chemical and structural changes for CA and HA2 electrodes in the $60-300^{\circ} \mathrm{C}$ and $360-630^{\circ} \mathrm{C}$ regions. This behavior is obviously more pronounced than in the case of NA and HA1 electrodes.

In figure 2, loss of mass (DTG curves) during heating over a large range of temperature $\left(20-800^{\circ} \mathrm{C}\right)$ is shown for all electrode types. Larger losses of mass for electrodes with additives indicate greater carbonization. CA and HA2 electrodes indicate two different technological procedures (cold addition vs hot addition). Similar results for these two cases allow the choice of a convenient commercial alternative.

In figure 3 , the average values of the major properties (pore volume, specific surface area, apparent density and porosity) are plotted for all cases. These values are better for electrodes without additives.

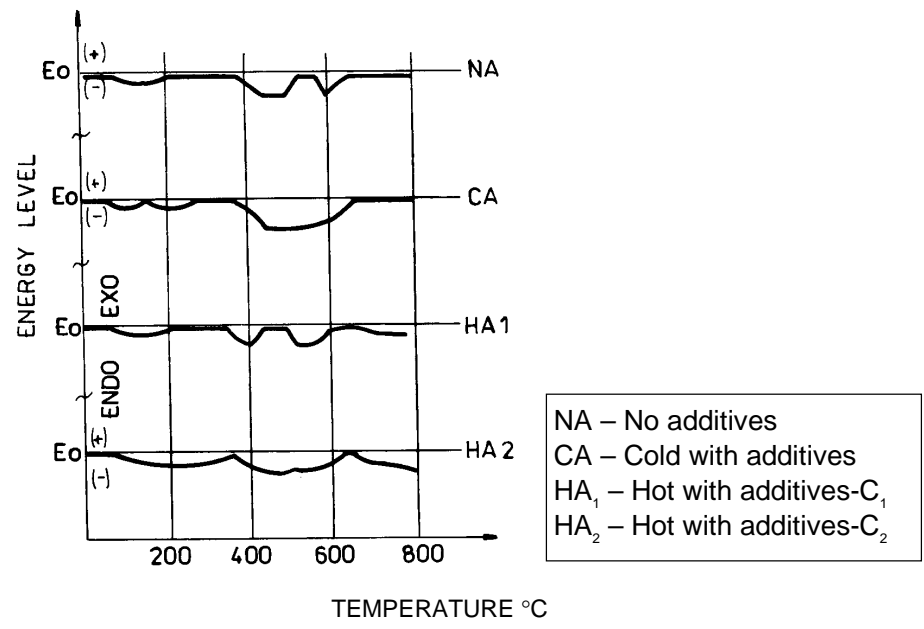

Figure 1. ATD diagrams.

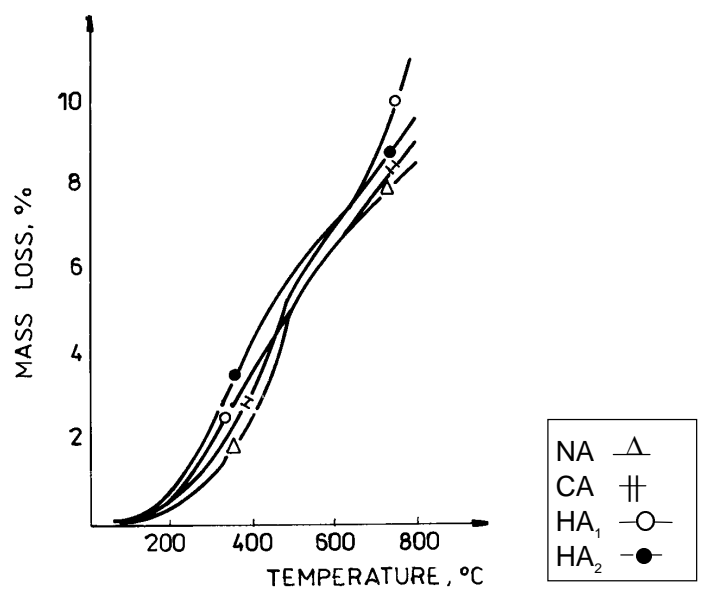

Figure 2. TDG diagrams. 


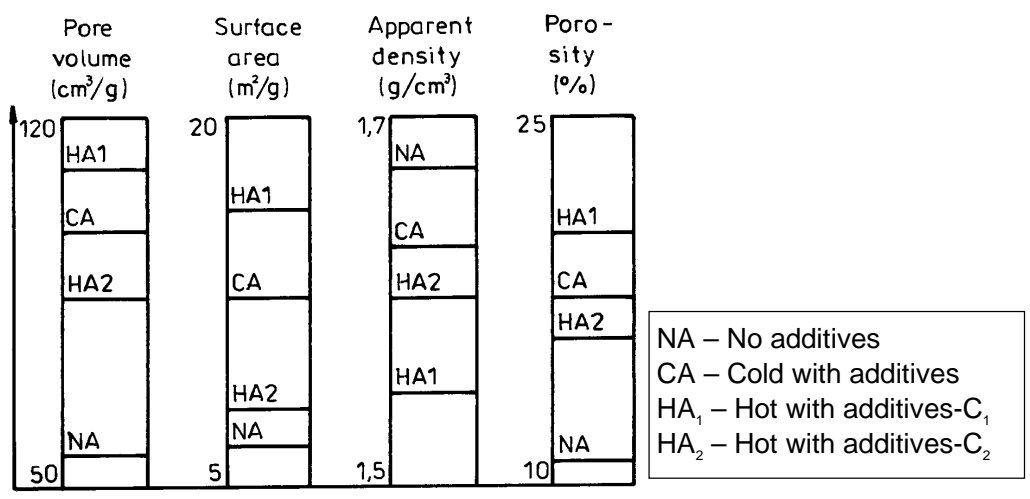

Figure 3. Green electrode.

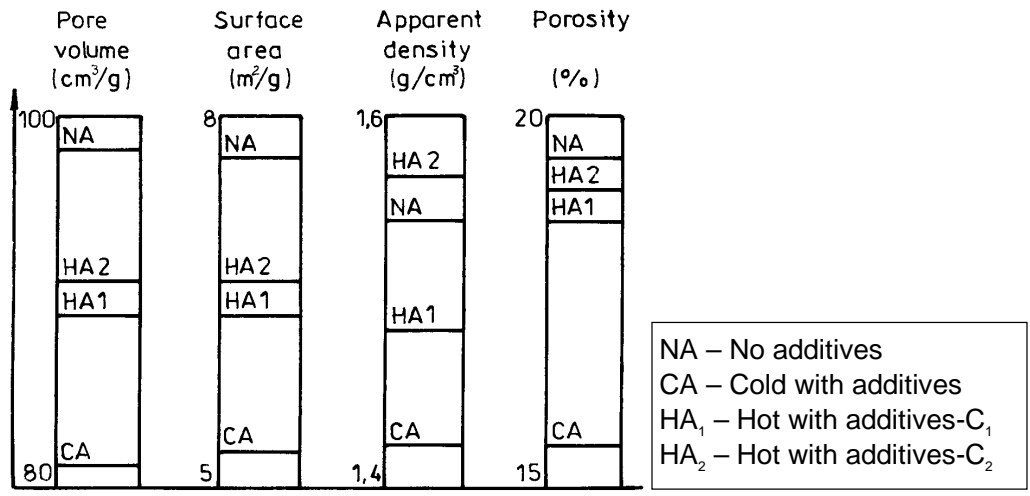

Figure 4. Baked electrode .

\subsection{Baked electrodes}

In figure 4 the same values for the major properties as in figure 3 are displayed on a comparable scale.

This step shows the actual interaction between additive and carbonaceous material, electrodes with additives being at a great advantage over electrodes without (for instance, porosity for the CA electrode is $20 \%$ less than for the NA case). It is important to note that average values for these properties are calculated as numerical averages, considering all three zones on a transversal plane, i.e. core, medium and external zones.

At this stage we can conclude that better carbon electrodes for the aluminium industry can be made.

According to pore size division in three regions (micropore, macropore and supermacropore), the study revealed the following conclusions regarding relative values for pore volume and specific surface area (table 1):

(a) For the micropore domain, the relative values for the NA electrode are bigger than for electrodes with additives, suggesting a better packed structure in the second case; 
Table 1. Baked electrodes - Relative sizes depend on pore size distribution. All volumes and areas are percentages (\%).

\begin{tabular}{|c|c|c|c|c|c|c|}
\hline \multirow[b]{3}{*}{ Type } & \multicolumn{6}{|c|}{ Pore size distribution } \\
\hline & \multicolumn{2}{|c|}{$0-300 \AA$} & \multicolumn{2}{|c|}{$300-10^{5} \AA$} & \multicolumn{2}{|c|}{$1.10^{5}-10^{6} \AA$} \\
\hline & $\begin{array}{c}\text { Relative } \\
\text { pore volume }\end{array}$ & $\begin{array}{c}\text { Relative } \\
\text { surface area }\end{array}$ & $\begin{array}{c}\text { Relative } \\
\text { pore volume }\end{array}$ & $\begin{array}{c}\text { Relative } \\
\text { surface area }\end{array}$ & $\begin{array}{c}\text { Relative } \\
\text { pore volume }\end{array}$ & $\begin{array}{c}\text { Relative } \\
\text { surface area }\end{array}$ \\
\hline NA & $20 \cdot 1$ & $93 \cdot 26$ & $65 \cdot 43$ & $6 \cdot 72$ & $14 \cdot 47$ & $0 \cdot 02$ \\
\hline CA & $17 \cdot 89$ & $90 \cdot 31$ & $63 \cdot 75$ & $9 \cdot 66$ & $18 \cdot 36$ & 0.03 \\
\hline HA1 & $19 \cdot 15$ & $90 \cdot 21$ & $67 \cdot 82$ & $9 \cdot 76$ & $13 \cdot 0$ & $0 \cdot 03$ \\
\hline HA2 & $17 \cdot 8$ & $91 \cdot 27$ & $71 \cdot 66$ & $8 \cdot 61$ & $10 \cdot 54$ & $0 \cdot 02$ \\
\hline
\end{tabular}

Table 2. Graphitized electrodes - Relative sizes depend on pore size distribution. All volumes and areas are percentages (\%).

\begin{tabular}{|c|c|c|c|c|c|c|}
\hline \multirow[b]{3}{*}{ Type } & \multicolumn{6}{|c|}{ Pore size distribution } \\
\hline & \multicolumn{2}{|c|}{$0-300 \AA$} & \multicolumn{2}{|c|}{$300-10^{5} \AA$} & \multicolumn{2}{|c|}{$1.10^{5}-10^{6} \AA$} \\
\hline & $\begin{array}{c}\text { Relative } \\
\text { pore volume }\end{array}$ & $\begin{array}{c}\text { Relative } \\
\text { surface area }\end{array}$ & $\begin{array}{c}\text { Relative } \\
\text { pore volume }\end{array}$ & $\begin{array}{c}\text { Relative } \\
\text { surface area }\end{array}$ & $\begin{array}{c}\text { Relative } \\
\text { pore volume }\end{array}$ & $\begin{array}{c}\text { Relative } \\
\text { surface area }\end{array}$ \\
\hline NA & $15 \cdot 17$ & $85 \cdot 24$ & 63.93 & 14.7 & $20 \cdot 9$ & 0.06 \\
\hline $\mathrm{CA}$ & $30 \cdot 0$ & $92 \cdot 93$ & $48 \cdot 13$ & $6 \cdot 98$ & $21 \cdot 87$ & 0.03 \\
\hline HA1 & $36 \cdot 05$ & $95 \cdot 65$ & $46 \cdot 39$ & $4 \cdot 33$ & $17 \cdot 56$ & 0.02 \\
\hline HA2 & $33 \cdot 12$ & $92 \cdot 61$ & $42 \cdot 94$ & $7 \cdot 31$ & $23 \cdot 94$ & 0.08 \\
\hline
\end{tabular}

(b) Electrodes with additives provide better conditions for subsequent impregnation processes, due to increased relative values for pore volume and specific surface area corresponding to macropores and supermacropores.

\subsection{Graphite electrodes}

Analyses of recorded data for the finished electrodes show major changes regarding the distribution of pore volume and specific surface area for the three defined regions of pore size (table 2).

The following conclusions can thus be drawn.

(a) Obvious modifications of the texture of electrodes with additives compared to electrodes without additives.

(b) Major contribution to specific surface area from micropores, this contribution being much bigger for electrodes with additives.

(c) In the micropore region, the relative pore volume for NA electrodes is $50 \%$ of corresponding levels for electrodes with additives.

(d) In the macropore and macro- and supermacropore regions as a whole, the relative values for pore volume and specific surface area are smaller for electrodes with additives. In agreement with these values, better performance in the impregnation step is determined by improved technology in the addition of the pitches. 
(e) It is known that the surface of pores with diameters greater than one micron may be accessible to oxidation and that the surface of pores with diameters of 1 to 10 microns is particularly significant in reducing electrode efficiency. Electrode performance can be increased by reducing the extent of oxidant-accessible surface and specific surface reactivity of pores in the 1 to 10 micron $(10,000 \AA-100,000 \AA)$ size range.

From the data shown in table 2, electrodes with additives have less oxidant-accessible surface area compared to NA electrodes, in the above mentioned range. This feature permits smaller consumption indices in steel electric arc furnaces.

The overall analysis shows that electrodes with additives are superior to NA electrodes as below:

(a) The coefficient of thermal expansion is lowered by $5-12 \%$;

(b) Traction strength increases by $30-40 \%$;

(c) Bending strength increases by $8-11 \%$;

(d) Resistivity increases by $15-20 \%$.

\section{Conclusions}

The proposed improvements in technology help us to obtain carbon and graphite electrodes with better characteristics (coefficient of thermal expansion, mechanical strength, porosity etc.). The changes in texture (pore size and pore volume distributions) show clear improvement in physico-chemical, mechanical and electric properties in different types of electrodes.

\section{References}

1. Honda H 1979 Efficient utilization of petroleum residues. In Proc. 10th World Petroleum Congress (Bucharest: Heyden \& Son)

2. Honda H 1980 J. Jpn. Petrol. Inst. 231

3. Cealih E F 1981 Baking of electrodes (Moscow: Metalurgia)

4. Iutes-Petrescu N 1996 Rheological properties of the hydrocarbons with great molecular weight, $\mathrm{Ph} \mathrm{D}$ thesis, Bucharest University, Bucharest 